\title{
What Can GLAST Say About the Origin of Cosmic Rays in Other Galaxies
}

\author{
Seth Digel $^{\mathrm{a}, \mathrm{b}}$, Igor V. Moskalenko ${ }^{\mathrm{a}, \mathrm{c}}$, and Jonathan F. Ormes, \\ P. Sreekumar ${ }^{d,}$ and P. Roger Williamson ${ }^{\mathrm{e}}$, \\ ${ }^{2}$ NASA/Goddard Space Flight Center, Greenbelt, Maryland \\ 'Universities Space Research Association, \\ 'National Research Council and Institute for Nuclear Physics, \\ Moscow State University \\ ${ }^{\mathrm{d}}$ Indian Space Research Organization, ${ }^{\mathrm{e}}$ Stanford University \\ on behalf of the GLAST collaboration
}

Presented at ACE 2000: The Acceleration and Transport of Energetic Particles Observed in the Heliosphere, 1/5/2000-1/8/2000, Indian Wells, CA, USA 


\title{
What Can GLAST Say About the Origin of Cosmic Rays in Other Galaxies?
}

\author{
Seth W. Digel ${ }^{\mathrm{a}, \mathrm{b}}$, Igor V. Moskalenko ${ }^{\mathrm{a}, \mathrm{c}}$, Jonathan F. Ormes ${ }^{\mathrm{a}}$, \\ P. Sreekumard ${ }^{\mathrm{d}}$, and P. Roger Williamson ${ }^{\mathrm{e}}$, \\ on behalf of the GLAST collaboration \\ ${ }^{a}$ NASA/Goddard Space Flight Center, Greenbelt, Maryland, ${ }^{b}$ Universities Space Research Association, \\ 'National Research Council and Institute for Nuclear Physics, Moscow State University, \\ ${ }^{d}$ Indian Space Research Organization, "Stanford University
}

\begin{abstract}
Gamma rays in the band from $20 \mathrm{MeV}$ to $300 \mathrm{GeV}$, used in combination with data from radio and Xray bands, provide a powerful tool for studying the origin of cosmic rays in our sister galaxies Andromeda and the Magellanic Clouds. Gamma-ray Large Area Space Telescope (GLAST) will spatially resolve these galaxies and measure the spectrum and intensity of diffuse gamma radiation from the collisions of cosmic rays with gas and dust in them. Observations of Andromeda will give an external perspective on a spiral galaxy like the Milky Way. Observations of the Magellanic Clouds will permit a study of cosmic rays in dwarf irregular galaxies, where the confinement is certainly different and the massive star formation rate is much greater.
\end{abstract}

\section{INTRODUCTION}

High-energy gamma rays are produced in interactions of high-energy cosmic rays with interstellar matter and photons. From the resulting diffuse emission of gamma rays, the properties of the cosmic rays can be inferred (e.g., (5)). Gamma rays have proven to be a useful probe of cosmic rays in the Milky Way, but gamma-ray telescopes to date have lacked the sensitivity and angular resolution to permit the same kind of detailed study of cosmic rays in external galaxics.

The Gamma-ray Large Area Space Telescope (GLAST) is the next generation high-energy (20 MeV$300 \mathrm{GeV}$ ) gamma-ray astronomy mission. It is part of the strategic plan of NASA's Office of Space Science and is currently planned for launch in 2005. GLAST will have a factor of 30 greater sensitivity than the Energetic Gamma-Ray Experiment Telescope (EGRET), launched in 1991 on the Compton Gamma-Ray Observatory. Derived performance parameters for our proposed design for the GLAST instrument, which was selected by NASA in February 2000, are presented in Table 1 and Figure 1. See the companion paper by Ormes et al. for information about the design and instrumental response of GLAST, and the Web site http://glast.gsfc.nasa.gov for information about the mission.
Table 1. Selected Parameters for GLAST and EGRET

\begin{tabular}{|c|c|c|}
\hline & EGRET & GLAST \\
\hline Energy Range & $0.02-30 \mathrm{GeV}$ & $0.02-300 \mathrm{GeV}$ \\
\hline Field of View & $0.5 \mathrm{sr}$ & $2.4 \mathrm{sr}$ \\
\hline Peak Eff. Area & $1500 \mathrm{~cm}^{2}$ & $13,000 \mathrm{~cm}^{2}$ \\
\hline \multicolumn{3}{|l|}{ Point Source } \\
\hline Sensitivity* & 5 & 0.16 \\
\hline Source Location $^{\dagger}$ & $5^{\prime}-90^{\prime}$ & $0.2^{\prime}-1^{\prime}$ \\
\hline Mission Life & & $\begin{array}{l}5 \text { years } \\
\text { (10-year goal) }\end{array}$ \\
\hline
\end{tabular}

* Sensitivity at high latitude after a 2-year survey for a 5- $\sigma$ detection, units $10^{-8} \mathrm{~cm}^{-2} \mathrm{~s}^{-1}$, for $E>100 \mathrm{MeV}$.

"Diameter of $95 \%$ confidence region; range: bright sources to sources of flux $10^{-8} \mathrm{~cm}^{-2} \mathrm{~s}^{-1}(E>100 \mathrm{MeV})$.

\section{ADVANCES WITH GLAST}

The only external galaxy that EGRET detected in the light of its interstellar gamma-ray emission was the Large Magellanic Cloud (LMC), which was not spatially resolved (10). GLAST will be able to map the diffuse gamma-ray emision of the LMC, as well as the fainter Small Magellanic Cloud (SMC) and Andromeda (M31) galaxies. 

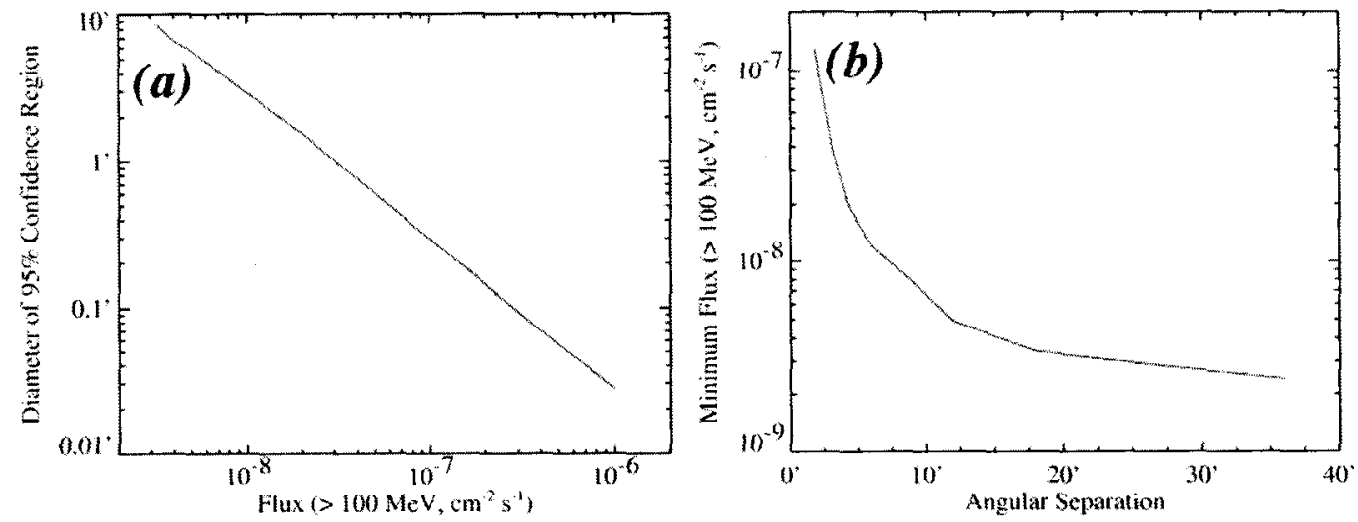

FIGURE 1. Expected performance of GLAST for localizing and resolving point sources. (a) Source localization at high latitudes. The position uncertainties for the brightest sources likely will be limited to $10-20^{\prime \prime}$ by uncertainty in spacecraft pointing and instrument alignment. (b) Minimum flux required to resolve two closely-spaced sources of equal flux. For both figures, the sources are assumed to have $E^{-2}$ photon spectra and to be observed at high latitudes in a one-year sky survey.

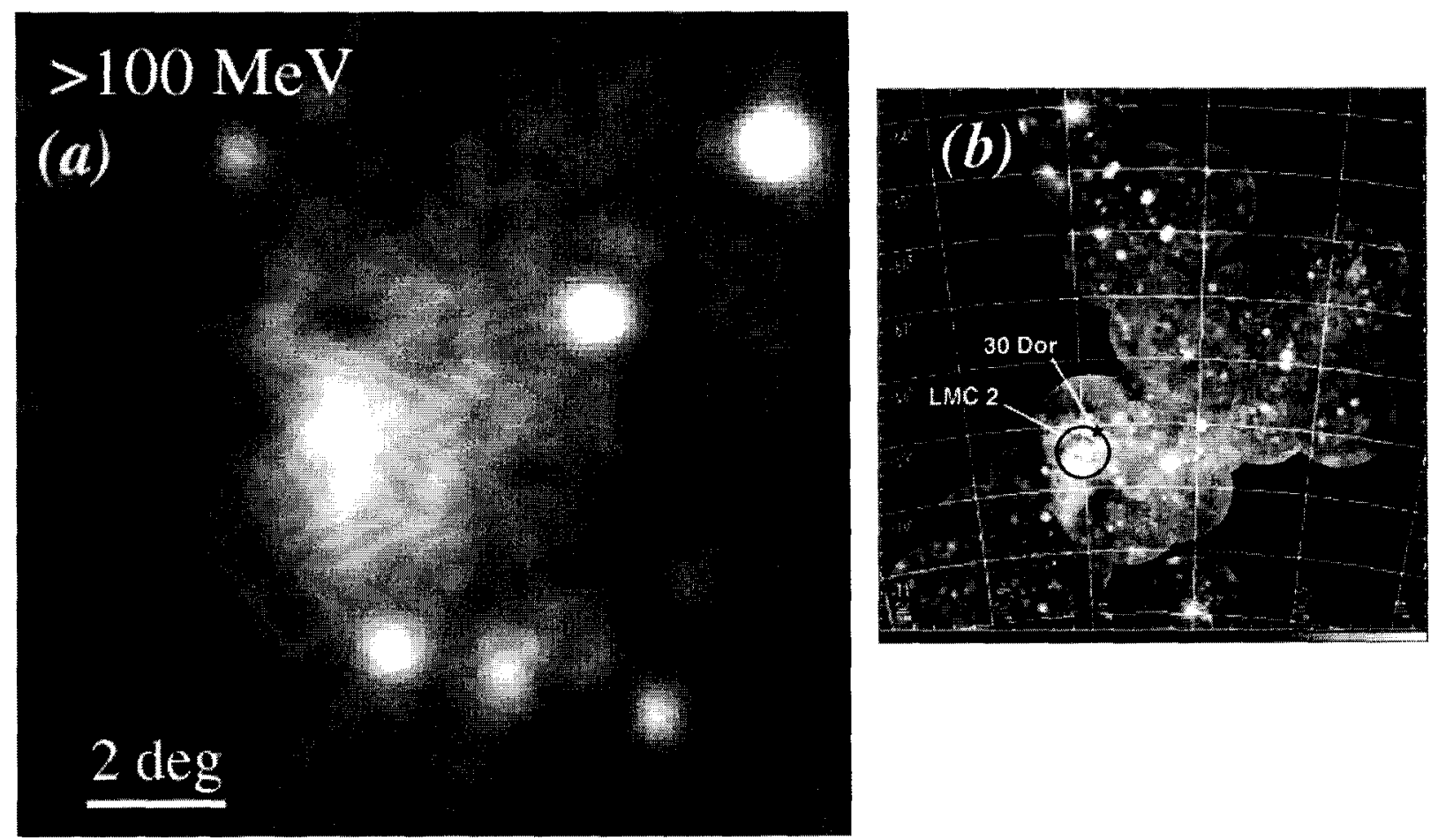

FIGURE 2. (a) Simulated map of the LMC in gamma rays (> $100 \mathrm{MeV}$ ) from a two-year sky survey with GLAST. The simulation is based on a model of the LMC by Sreekumar (12) and also includes foreground diffuse emission from the Milky Way and an isotropic background consisting of a distribution of faint point sources. (b) The LMC in 3/4-keV X-rays, from a mosaic of pointed observations with ROSAT (8). The intense emission regions of 30 Doradus and LMC superbubble 2 are indicated.

\section{$L M C$}

The LMC will be well-resolved by GLAST (Fig. 2). The cosmic-ray distribution can be studied in detail by analyzing the gamma-ray data together with $21-\mathrm{cm} \mathrm{H} \mathrm{I}$ and $2.6-\mathrm{mm} \mathrm{CO}$ surveys of the interstellar medium of the galaxy (see, e.g., (2)). GLAST data should reveal the degree of enhancement of cosmic-ray density in the vicinity of the massive star-forming region 30 Dor and the associated superbubble LMC 2 (6); see the X-ray image in Fig. 2. The LMC 2 superbubble is among the largest of several prominent superbubbles in the LMC, 


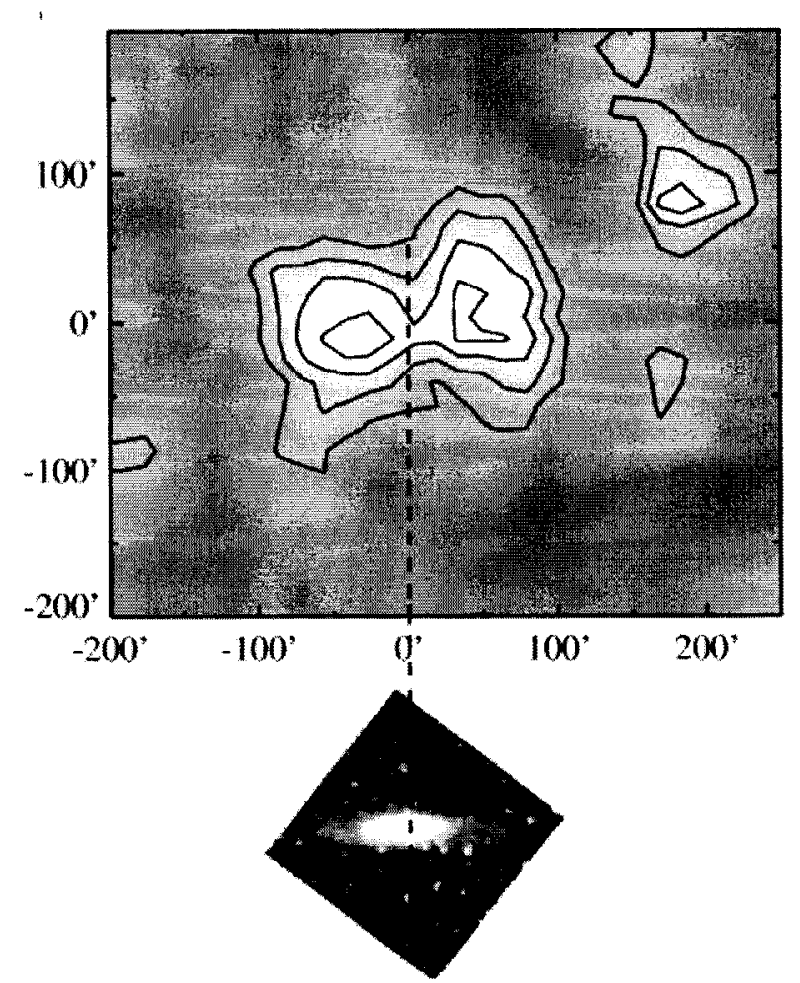

FIGURE 3. Simulated map of M31 from a five-year sky survey with GLAST (approximately equivalent to 6 months of observations with $\mathrm{M} 31$ within $30^{\circ}$ of the center of GLAST's field of view). The image shows gamma-rays with energies $>1 \mathrm{GeV}$, and has been smoothed to reduce statistical fluctuations. The simulated point source in the upper right indicates the angular resolution of the image, and the inset shows the location and extent of the optical disk of the galaxy. The diffuse emission was modelled based on the distribution of gas in M31, which extends much further than the optical disk, and the EGRET upper limit for the galaxy (1). Contours are spaced by $2 \times 10^{-7}$ $\mathrm{cm}^{-2} \mathrm{~s}^{-1} \mathrm{sr}^{-1}$ from $2.2 \times 10^{-6} \mathrm{~cm}^{-2} \mathrm{~s}^{-2} \mathrm{sr}^{-1}$.

most of which represent regions like 30 Dor that are further evolved. If superbubbles are sources of cosmic rays distinct from individual supernovas (e.g., (4)), superbubble 2 , which subtends $1^{\circ}$, may be marginally spatially resolved by GLAST. The extent to which the diffuse emission of the LMC can be attributed to unresolved gammaray pulsars has been considered by Hartmann et al. (3) and Zhang \& Cheng (14). The expectation is that the pulsar contribution could be significant, up to $35 \%$, for energies $>1 \mathrm{GeV}$. GLAST is unlikely to detect individual pulsars in the LMC, but may be able to address the question of the pulsar fraction with a sensitive measurement of the high-energy spectrum.

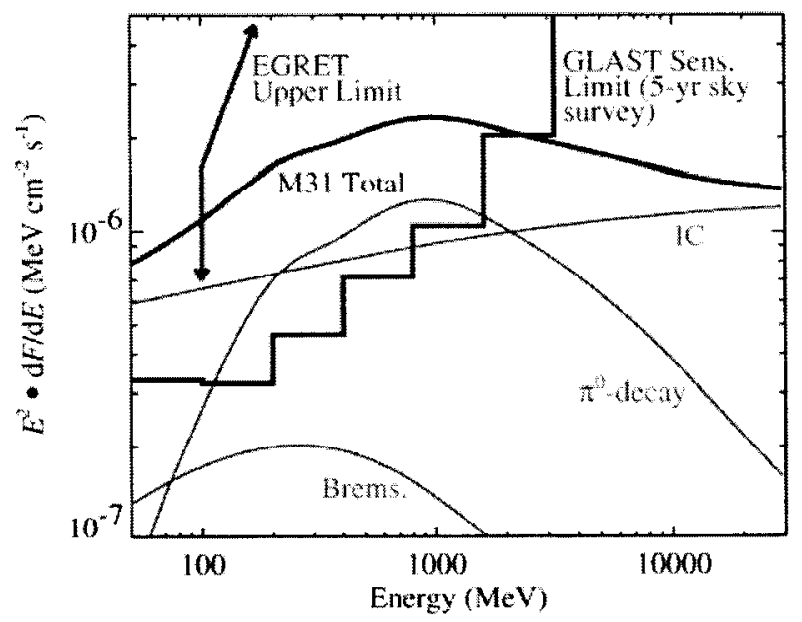

FIGURE 4. Simulated spectrum of M31, obtained by scaling the luminosity spectrum of the whole Milky Way derived by Strong et al. (13) (4-kpc halo model) to match the upper limit flux of Blom et al. (1) for M31. The differential flux sensitivity of GLAST for a five-year sky survey and the EGRET upper limit of Blom et al. are also shown, along with the individual components of the overall spectrum: inverse Compton, Bremsstrahlung, and $\pi^{0}$-decay.

\section{$S M C$}

Detection of the diffuse gamma-ray flux of the SMC by GLAST will be useful to verify conclusions about the galactic origin of cosmic rays (e.g., Sreekumar et al. (11) based on EGRET data). The non-detection of the SMC, with a 2- $\sigma$ upper limit of $5 \times 10^{-8} \mathrm{~cm}^{-2} \mathrm{~s}^{-1}(>100 \mathrm{MeV})$ led Sreekumar et al. to conclude that the most likely model for the distribution of cosmic rays in the SMC is one for which the galaxy is disintegrating and cosmic rays are only very poorly confined. In this circumstance, the predicted flux is $(2 \pm 3) \times 10^{-8} \mathrm{~cm}^{-2} \mathrm{~s}^{-1}(>100 \mathrm{MeV})$ (9), well within the reach of GLAST.

\section{M31}

The EGRET 2- $\sigma$ upper limit for the gamma-ray flux of M31 is $1.6 \times 10^{-8} \mathrm{~cm}^{-2} \mathrm{~s}^{-1}(>100 \mathrm{MeV})$, which is much less than the flux of the Milky Way at M31 (1). The cosmic-ray densities in M31 are certainly lower than in the Milky Way, and it has less ongoing massive star formation. At a flux level of $1 \times 10^{-8} \mathrm{~cm}^{-2} \mathrm{~s}^{-1}$ (>100 $\mathrm{MeV})$, GLAST will resolve the diffuse gamma-ray emission along the major axis of M31, to provide information about the relationship between cosmic rays, star formation rate, and interstellar gas on a large scale (Fig. 3). GLAST may also measure the distribution of cosmic rays 
in the halo of M31. Spectral measurements may allow a global assessment of inverse-Compton, electronBremsstrahlung, and $\pi^{0}$ decay contributions to the interstellar emission (Fig. 4). The unexplained "GeV excess" for the Milky Way (5) will also be detected if present in M31. From the gamma-ray spectra of the Milky Way and M31 the contribution of normal galaxics to the extragalactic gamma-ray background can begin to be assessed.

Detailed studies of M31 that will be possible with GLAST will benefit from further development of cosmicray models for the Milky Way, for which results from gamma-ray observations can be checked with direct observations of cosmic rays.

\section{CONCLUSIONS}

For the first time, GLAST will enable spatial and spectral studies of diffuse gamma rays from external galaxies. Diffuse, high-energy gamma rays are diagnostic of cosmic-ray densities, which especially for the proton component are difficult to determine from observations at other wavelengths. When considered together with Xray and radio observations, GLAST data promise a fairly complete understanding of the production, propagation, and confinement of cosmic rays in Local Group galaxies.

IVM acknowledges support from an NAS/NRC Senior Associateship.

\section{REFERENCES}

1. Blom, J. J., et al., Astrophys. J. 516, 44, (1999).

2. Cohen, R. S., et al., Astrophys. J. 331, L95, (1988).

3. Hartmann, D. H., Brown, L. E., and Schnepf, N., Astrophys. J. 408, L13, (1993).

4. Higdon, J. C., et al., Astrophys. J., 509, 33, (1998).

5. Hunter, S. D., et al., Astrophys. J. 481, 205, (1997).

6. Meaburn, J., MNRAS 192, 365, (1980).

7. Ormes, J. F., et al., these proceedings, (2000), astro-ph/0003270.

8. Snowden, S. L., and Petre, R., Astrophys. J. 436, L123, (1994).

9. Sreekumar, P., and Fichtel, C. E., Astron. and Astrophys. 251, 447, (1991).

10. Sreekumar, P., et al., Astrophys. J. 400, L67, (1992).

11. Sreekumar, P., et al., Phys. Rev. Lett. 70, 127, (1993).

12. Sreekumar, P., priv. comm., 1999.

13. Strong, A. W., Moskalenko, I., and Reimer, O., Astrophys. $J ., 537$, in press (2000), astro-ph/ 9811296 .

14. Zhang, L., and Cheng, K. S., MNRAS, 294, 729, (1998). 